\title{
The Influence of Memory on Perception: It's Not What Things Look Like, It's What You Call Them
}

\author{
Holger Mitterer \\ Max Planck Institute for Psycholinguistics
}

\author{
Jochen Müsseler
}

Aachen University

\author{
Jörn M. Horschig \\ Max Planck Institute for Psycholinguistics \\ and Maastricht University \\ Asifa Majid \\ Max Planck Institute for Psycholinguistics
}

\begin{abstract}
World knowledge influences how we perceive the world. This study shows that this influence is at least partly mediated by declarative memory. Dutch and German participants categorized hues from a yellow-to-orange continuum on stimuli that were prototypically orange or yellow and that were also associated with these color labels. Both groups gave more "yellow" responses if an ambiguous hue occurred on a prototypically yellow stimulus. The language groups were also tested on a stimulus (traffic light) that is associated with the label orange in Dutch and with the label yellow in German, even though the objective color is the same for both populations. Dutch observers categorized this stimulus as orange more often than German observers, in line with the assumption that declarative knowledge mediates the influence of world knowledge on color categorization.
\end{abstract}

Keywords: color perception, top-down effects, perceptual memory, conceptual memory

Observers tend to perceive the world in accordance with their expectations. A yellow-orange hue is more likely to be categorized as orange on a carrot than on a banana (Mitterer \& de Ruiter, 2008). A face is perceived to be lighter if it contains prototypical White features rather than Black ones (Levin \& Banaji, 2006). A speech sound that could be /t/ or /d/ is perceived as /d/ in _ash but as /t/ in _ask (Ganong, 1980; Warren, 1970). A slightly ambiguous letter-such as an $H$ in which the two vertical lines are tilted inward-is perceived as $A$ if it occurs in $C_{-} T$ but as $H$ if it occurs in $T_{-} E$ (McClelland \& Rumelhart, 1981). These object-based biases are useful for the observer, because perceptual evidence in the real world tends to be less than perfect. The light reflected from a banana depends strongly on the lighting source; speech sounds can be masked by ambient noise; and careless handwriting can blur the distinctions between different letters. When faced with ambiguity, the observer is going to be right more often than wrong when assuming that a banana really is yellow-at least when in a supermarket.

There is a longstanding debate concerning whether such biases show a top-down flow of information. Undoubtedly, an interactive

Holger Mitterer and Asifa Majid, Max Planck Institute for Psycholinguistics, Nijmegen, The Netherlands; Jörn M. Horschig, Max Planck Institute for Psycholinguistics, Nijmegen, The Netherlands, and MICC, Department of Mathematics, Maastricht University, Maastricht, The Netherlands; Jochen Müsseler, Psychology Department, RWTH Aachen University, Aachen, Germany.

This project was part of an internship of the second author at the Max Planck Institute for Psycholinguistics.

Correspondence concerning this article should be addressed to Holger Mitterer, Max Planck Institute for Psycholinguistics, Wundtlaan 1, 6525 XD Nijmegen, The Netherlands. E-mail: Holger.Mitterer@mpi.nl activation and inhibition account elegantly explains how we perceive the world in line with world knowledge (McClelland \& Elman, 1986; McClelland \& Rumelhart, 1981). The two left panels in Figure 1 show such an architecture. Information from perception is fed forward to memory, and the content of memory then directly influences how the stimulus is perceived. Consider the _ash versus _ask example above. The words dash and task, respectively, fit the perceptual evidence best because tash and dask are not words in English. Lexical activation from the real words feeds back to the phoneme-perception units. This feedback then boosts activation for the /t/ unit when the input is_ask, which in turn inhibits the /d/ unit. As a consequence, $/ \mathrm{t} /$ is more strongly activated than $/ \mathrm{d} / \mathrm{in}$ response to the stimulus_ask. This explains why listeners hear a /t/ more often in_ask than in_ash. Such top-down feedback may help us overcome the immense variability that we face in perception, like the invariance problem in speech perception (Liberman, 1996) or the color-constancy problem in visual perception (Brainard, 2004).

Skeptics, however, have argued that feedback from a lexical to a prelexical level is not necessary to explain how world knowledge biases perceptual categorization (Massaro, 1998; Norris, McQueen, \& Cutler, 2000). According to this alternative account, when a listener is confronted with input such as _ask, he or she just has to weigh the perceptual evidence (which is ambiguous) against the lexical evidence (which indicates a / $t$ /) to arrive at the decision that there was a /t/ and not a /d/ in the input. That is, the perceptual evidence and the world knowledge are merged to arrive at a perceptual decision. This alternative model is depicted in the two right panels of Figure 1. Note that there is no direct feedback from memory on perceptual processes. In support of this view, Frauenfelder and Peeters (1998) found that top-down feedback is-in 


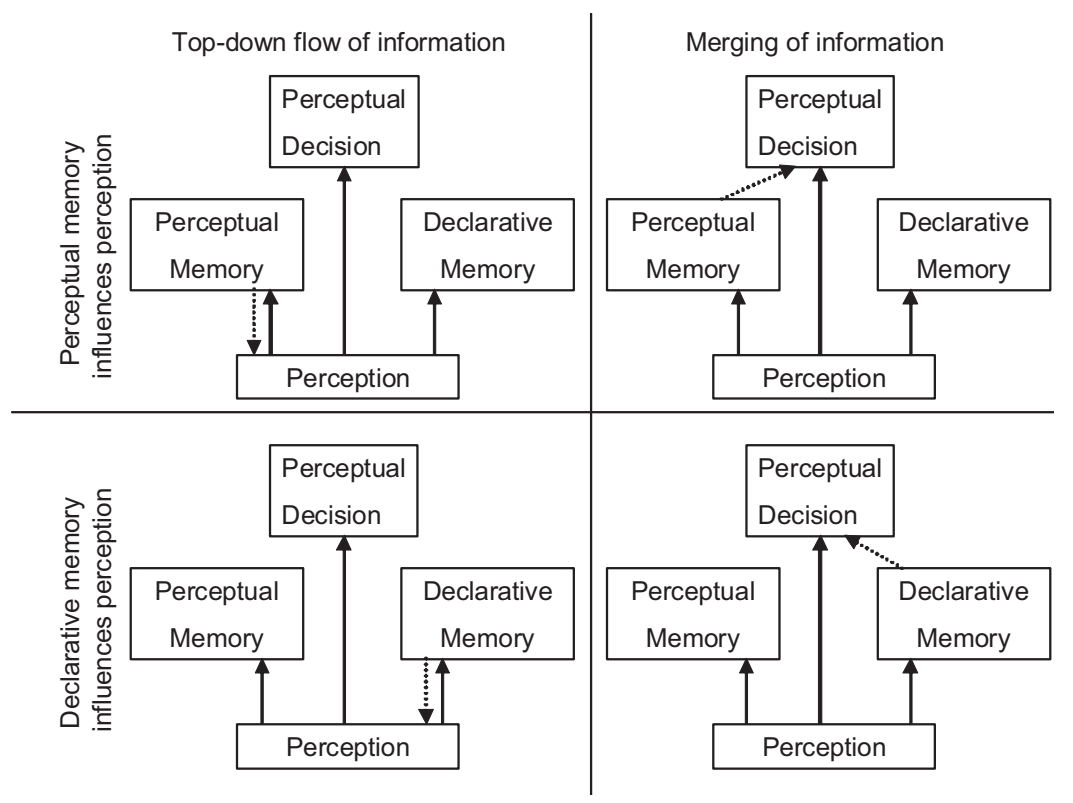

Figure 1. Four ways of accounting for the influence of memory on perception. The solid arrows show uncontroversial flow of information, and dotted arrows show controversial connections.

contrast to the general intuition-not a useful tool for perception to overcome the variability that perception faces. Frauenfelder and Peeters showed that an interaction-activation model of word recognition (TRACE model; McClelland \& Elman, 1986) works just as efficiently when top-down connections are removed.

To distinguish these two accounts-feedback to perceptual encoding versus merging at perceptual decision-recently researchers have moved to procedures in which recruitment of explicit knowledge is thought unlikely to be involved, thus potentially ruling out a merging account. For example, in color perception, Hansen, Olkkonen, Walter, and Gegenfurtner (2006) asked participants to change the hue of an object-by pressing one of four buttons that increased or decreased the hue values in a twodimensional color space-so that it appeared gray to them. In contrast to a simple categorization task ("Is this object yellow or orange?"), this task does not necessitate access to color labels. For objects without a prototypical color, participants performed quite well and found an objective achromatic hue. But for objects with a prototypical color, participants chose a hue that was opposite to the object's natural hue, so that the color of a banana was changed to a slight bluish-gray instead of a truly achromatic gray hue. This indicates that a banana that is objectively gray actually appears slightly yellow. Although this task does not necessitate recruitment of color labels, it is possible that they are nonetheless activated. It appears that activation of object concepts also activates knowledge of their color. For example, hearing the word banana activates the concept "yellow" (Huettig \& Altmann, 2004). Likewise, it is probable that when a person sees a banana, the concept "yellow" is also activated. So it is quite possible that declarative knowledge about object color influenced perceptual decision making without influencing perceptual encoding.

A different sort of indirect task has been developed in speechperception research. Phonetic research has shown that in compar- ison with an unambiguous /sh/, an unambiguous /s/ makes a following stop sound more like /k/ (rather than /t/; Mann \& Repp, 1981). In addition, an ambiguous sound between /s/ and /sh/ is perceived as $/ \mathrm{sh} /$ in fooli_and as $/ \mathrm{s} /$ in christma_. So the question becomes whether lexically induced percepts of /s/ and /sh/ influence the perception of a following stop as either $/ \mathrm{t} / \mathrm{or} / \mathrm{k} / \mathrm{just}$ as the signal-based percepts of unambiguous $/ \mathrm{sh} /$ and $/ \mathrm{s} / \mathrm{do}$. This would be predicted by a feedback to perceptual encoding account. Although there have been some positive findings (Elman \& McClelland, 1988; Magnuson, McMurray, Tanenhaus, \& Aslin, 2003; Samuel \& Pitt, 2003), there is still some doubt that these findings show top-down feedback (McQueen, 2003; McQueen, Jesse, \& Norris, 2009; Mitterer, 2007).

The studies summarized above - and in fact most of the existing literature- have focused on questions about information flowthat is, whether higher level information directly influences perceptual processing. There is, however, a related-though logically separate-issue: What kind of information mediates such objectbased biases? Or to put it another way, is the influence of world knowledge on perceptual categorization mediated by declarative or perceptual memory? It has long been accepted that human memory is not a unitary system but that there are different forms of memory, including perceptual memory about how things look and sound, and declarative memory, such as the knowledge that bananas are called "yellow" (e.g., Blaxton, 1999; Squire, 2004). The question we address is which of these two memory systems is implicated in object-based biases. In the _ask example, is it abstract phonological knowledge that can be consciously entertained (e.g., that the word task starts with the same phoneme as the word town), or is it the perceptual knowledge about what type of sound precedes _ask — that is, knowledge that this sound tends to be a high-frequency (in Hertz), high-amplitude burst. In the color domain, does a banana appear more yellow than it actually is because 
we know (declaratively) that "bananas are yellow," as depicted in the two lower panels of Figure 1? Or do we assume that the hue on this banana is probably similar to the hues we (perceptually) remember from encounters with other bananas, as depicted in the upper panels of Figure 1?

The conundrum is that these two potential factors-declarative and perceptual memory-are (nearly) perfectly correlated: The perceptual memories of typical bananas correlate with the verbal label that participants use to describe bananas. This is probably why—as far as we are aware-previous research has not attempted to tease apart the influence of these different forms of memory on perception. Nevertheless, we identified a situation in which the two factors can be separated: traffic lights in Germany and the Netherlands. The topmost and bottommost colors of traffic lights are described with the cognate color terms for green and red (German: grün and rot; Dutch: groen and rood), but the middle light is subject to variation: Germans use their equivalent of yellow (i.e., gelb), whereas the Dutch use the term for their color orange (i.e., oranje). This diversity arises even though the perceptual memories (at least of typical undergraduates) must be similar, because traffic lights in the European Union are bound by a European norm (EN 12368, Deutsches Institut für Normierung, 2006). This situation allows us to tease apart the contributions of perceptual memory and verbal labels on perceptual decisions.

In line with previous work investigating the influence of world knowledge on perception, we created a continuum of hues ranging from a good yellow to a good orange (Ganong, 1980; Levin \& Banaji, 2006; Pitt \& McQueen, 1998; Samuel \& Pitt, 2003). These hues were then used to color the middle light of a traffic light, and observers were asked whether the hue was yellow or orange. We hypothesized that if German participants call the hues on the middle traffic light "yellow" more often than Dutch participants, then the object bias in color perception stems from declarative knowledge, because Germans call this object gelb (English: yellow), whereas the Dutch call it oranje (English: orange). If, however, perceptual memory of traffic lights induces the object bias in color perception, the two groups should not differ in their "yellow" responses, as they have similar exposure to traffic lights.

There is a caveat to this prediction. The color categories of Dutch and German may not be identical, even though nearly all color terms are cognate in the two languages. Several recent articles have shown that color perception and processing are not completely determined by (physiological) universals (Roberson, Davies, \& Davidoff, 2000; Winawer et al., 2007). So a difference between Dutch and German observers in the number of "yellow" responses to hues displayed on a traffic light would not necessarily indicate different labeling of the traffic light colors but could be attributable to a difference in the color categories of yellow (Dutch: geel, German: gelb) and orange (Dutch: oranje, German: orange) instead. To forestall this possibility, we included a colorneutral stimulus (a sock) that was colored in the same hues as the traffic light. If the color categories differ between the groups for our stimuli, this should lead to a group difference for both the sock and the traffic light stimulus. On the other hand, if a group difference is observed only for hues on the traffic light stimulus, a misalignment between Dutch and German yellow and orange categories cannot account for the data.

In addition to the sock, we included a prototypically orange stimulus (a carrot) and a prototypically yellow stimulus (a banana) to replicate the object bias effect in color categorization. If both Dutch and German participants give equivalent "yellow" responses to the traffic light and both groups give more "yellow" responses to the banana than to the carrot, we have strong evidence that the object bias in color categorization is caused by perceptual memory. If instead Dutch and German participants give different color responses to the traffic light, then the object bias in color categorization is (at least in part) due to declarative memory.

\section{Method}

\section{Participants}

Thirty-nine volunteers participated in the study. All had normal or corrected-to-normal vision and reported no color perception impairments. Twenty were native speakers of German studying at the RWTH Aachen University, Germany. Nineteen were native speakers of Dutch from the subject pool of the Max Planck Institute for Psycholinguistics, the Netherlands.

\section{Materials and Procedure}

All experiments were run in a completely darkened room with a 15-in. LCD monitor (Iiyama TXA3823MT) as the only source of light. We generated a continuum of six hues from yellow to orange. The Commission internationale de l'éclairage (CIE) XYZ coordinates of these hues were as follows: Hue 1: [60.0, 53.8, 8.5]; Hue 2: [61.4, 57.4, 9.0]; Hue 3: [63.9, 60.3, 9.4]; Hue 4: [65.7, 64.3, 10.2]; Hue 5: [67.6, 67.6, 10.8]; Hue 6: [69.8, 71.5, 11.5]. These hues were then placed on four different pictures (see Figure 2): a traffic light with the middle light being "on," a sock, a banana, and a carrot. These four pictures and six hues led to 24 experimental stimuli. These stimuli were presented to the participants with the Presentation software (Version 11.26).
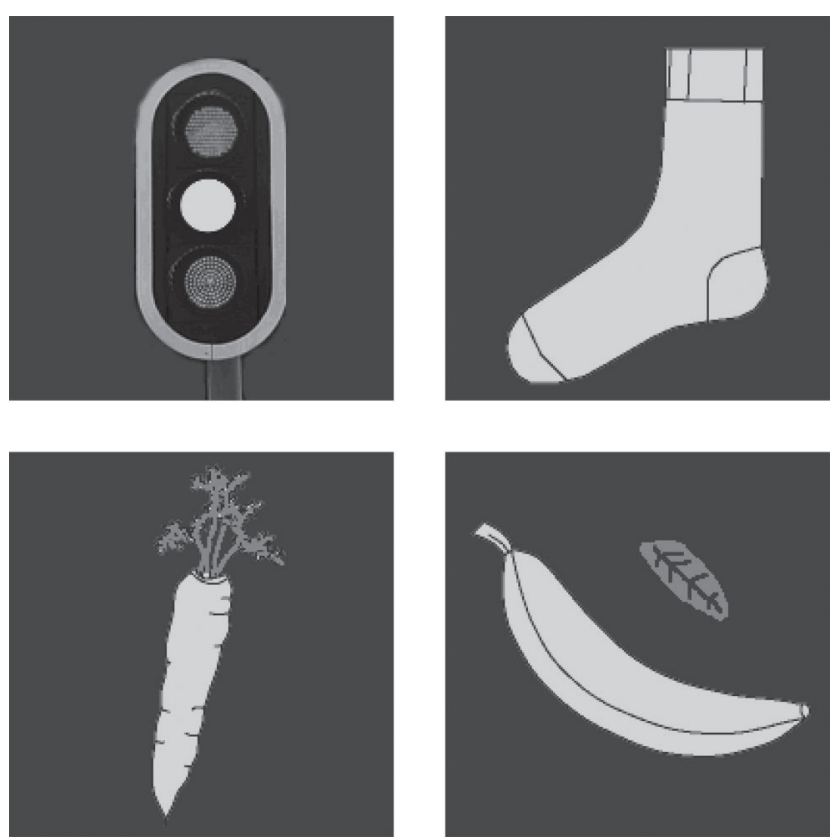

Figure 2. The four stimuli used in this experiment. 
Participants were tested individually, facing the computer screen. They were instructed to categorize the color on the objects as "yellow" or "orange" by pressing the corresponding response button. They were then presented with 15 blocks in which the 24 experimental stimuli were each presented once. Within each block, the stimuli were randomly permutated.

\section{Design and Analysis}

In this experiment, we tested the extent to which the proportion of "yellow" responses was influenced by the hue, the object identity, and the native language of the observer. Given the categorical nature of the dependent variable, we used a linear mixedeffect model with a logistic linking function (as suggested by Jaeger, 2008) to predict the proportion of "yellow" responses, with the factors hue, object, and native language of the observer as fixed effects and observer as a random effect.

\section{Results}

Figure 3 presents the results as the percentage of "yellow" responses. There was a tendency for the Dutch participants to give more "yellow" responses to all objects but the traffic light. The statistical analysis accordingly shows-besides the trivial effect of hue-an overall interaction of Object $\times$ Native Language, $F(3$, $13939)=8.59, p<.001 .{ }^{1}$ We therefore ran separate analyses for each object; the analyses showed no effect of native language for the objects carrot, banana, and sock $(p>.1)$. There was, however, a significant effect of native language for the traffic light, $b_{\text {language }}=$ Dutch $=0.51, p<.05$. We examined whether the influence of native language differed between the steps by introducing a Hue $\times$ Native Language interaction, which was not significant $(p>.1)$. This may seem surprising, given the small difference for the most yellowish hues, but it is important to
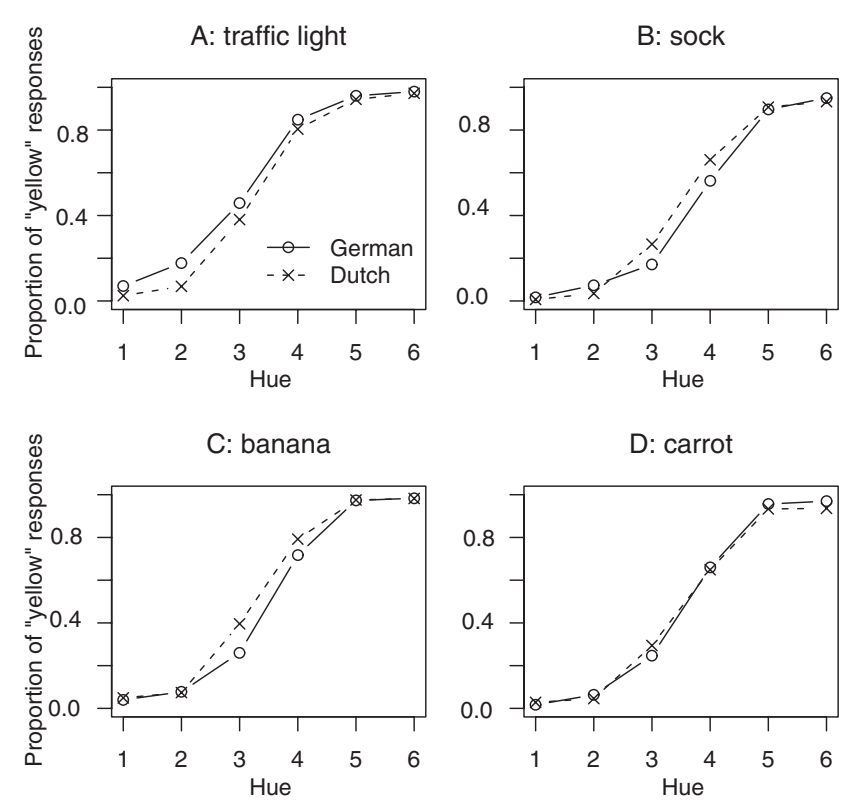

Figure 3. The proportion of "yellow" responses for the two groups of participants, depending on the hue and the presented objects. remember that small differences near the margins of a proportional scale are (correctly) inflated by the logistic-regression method (cf. Jaeger, 2008).

Disregarding the language variable, we found that there were more "yellow" responses across the hues if the hues were on the banana than if the hues were on the carrot, $b_{\text {object }}=$ banana $=0.47$, $p<.001$, and that there were more "orange" responses if the hue was on the sock than if it was on the carrot, $b_{\text {object }}=$ sock $=0.29$, $p<.001$.

\section{Discussion}

In this experiment, we tested whether the influence of world knowledge on perceptual categorization is caused by declarative or perceptual memory. It was possible to tease these factors apart because Dutch and German observers differ in their declarative, but not perceptual, memory for traffic lights: Traffic lights are bound to European regulations in both countries, but the Dutch call the middle light oranje (orange), whereas the Germans call it gelb (yellow). German observers categorized hues applied to the middle traffic light as "yellow" more often than Dutch observers did, whereas there were no significant differences between the groups in color categorization for other objects. The difference in declarative memory for traffic lights is the most likely cause of this result.

There were two additional findings, one expected and one unexpected, that are independent of native language. Both Dutch and German participants gave more "yellow" responses for a banana than for a carrot, in line with the world knowledge that "bananas are yellow" and "carrots are orange." It was unexpected, however, that the sock object induced even more "orange" responses than the carrot. One explanation for this finding is a possible difference in simultaneous brightness contrast. Yellow is in general a brighter color than orange. The objects were displayed on a dark gray background, which made their boundaries appear brighter. Because the sock was the largest object in the line drawings we used (see Figure 2), this contrast contributed a little less to its overall appearance than it did for the slender carrot, which may therefore have appeared brighter and more yellow than the sock.

However, this possible effect of simultaneous contrast cannot be the cause of the main result of this experiment: the difference in "yellow" responses between Dutch and German observers for the traffic light stimulus. The picture was, after all, the same for both groups of observers. Could there be yet another explanation for the observed difference between the groups? One possibility is that color boundaries between yellow and orange differ between the two groups. We ruled out this possibility by including non-trafficlight objects. Any differences in color boundaries would have influenced the responses on the other objects as well, but there we did not find a difference between the two groups.

\footnotetext{
${ }^{1}$ As the large number of degrees of freedom indicates, the statistical method uses every single data point (39 participants $\times 24$ pictures $\times 15$ presentations). It is, however, not a simple logistic regression, which would fail to take into account the fact that some of the data points are not independent (i.e., those coming from the same participant). Baayen, Davidson, and Bates (2008) showed that despite the large number of degrees of freedom, the mixed-effect models are not prone to yield Type I errors.
} 
The results seem to indicate that declarative knowledge and not perceptual knowledge leads us to perceive the world in line with our expectations. This is consistent with previous findings suggesting that the perception of color relies on actively naming these colors: Participants judge two colors from the same linguistic category to be more similar to one another than stimuli from different linguistic categories (a categorical perception effect). Crucially, when the boundaries of color categories vary across languages, performance is determined by language-specific categories rather than by perceptual distance (e.g., Roberson et al., 2000). Although these effects by themselves could be mediated by a language-induced, top-down reorganization of the perceptual space, several recent findings show that these effects may instead be mediated by an active recruitment of color labels. Categorical perception is stronger in the right visual field than in the left visual field (Drivonikou et al., 2007; Gilbert, Regier, Kay, \& Ivry, 2006; Roberson, Pak, \& Hanley, 2008). This implicates an active role for verbal labels, because the right visual field is projected to the left hemisphere, in which most lexical knowledge is stored. Moreover, under verbal-but not spatial-interference, categorical perception of color disappears (Gilbert et al., 2006; Roberson \& Davidoff, 2000; Winawer et al., 2007). Taken together, these findings suggest that declarative memory has a crucial role to play in perceptual decision making.

Obviously, we cannot rule out that perceptual memory has a role to play as well. Nevertheless, the current data indicate that this role may be rather minor. As it turns out, the effect of object identity (banana vs. carrot) is very similar in size to the effect of languagespecific labeling for the traffic light stimulus. Both effects are around $5 \%$ in the descriptive data, leading to a change of 0.5 in the $\log$ (odds) in the statistical model. Hence, a labeling bias is sufficient to explain the observed numerical difference in "yellow" responses to the banana and carrot.

We have argued that verbal labels mediate the influence of world knowledge on perceptual decision. In doing so, we contribute to the lively debate concerning the extent to which language can shape thinking and perception (Davidoff, 2001; Davidoff, Goldstein, \& Roberson, 2009; Franklin, Wright, \& Davies, 2009; Goldstein, Davidoff, \& Roberson, 2009; Kay \& Regier, 2006; Levinson, Kita, Haun, \& Rasch, 2002; Li \& Gleitman, 2002; Majid, Bowerman, Kita, Haun, \& Levinson, 2004). At first sight, our results are clearly encouraging for a Whorfian view by showing that simply calling an object "yellow" or "orange" influences how hues on these objects are categorized. This dovetails well with the other recent findings of color naming on color perception cited above, which also indicate that verbal labeling influences color perception. However, the Whorfian view often implicitly assumes an effect of language on perceptual encoding rather than on perception decisions. The merge model (Norris et al., 2000), which does not postulate an influence of world knowledge on perceptual encoding, is nevertheless sufficient to explain many Whorfian effects. Consider Winawer et al. (2007), who showed that Russian observers are faster to discriminate two hues if they are named differently. The merge architecture predicts this result, as the "different" response is suggested both by the perceptual evidence and by declarative knowledge, which leads to a faster response time than when declarative knowledge suggests a conflicting "same" response. If verbal interference prevents declarative knowledge from exerting an influence, only perceptual evidence drives responses and the effects of language-specific color categories should disappear, as they do in the empirical data.

When putting forward a merge-type architecture, we do not mean to trivialize the influence of language on perceptual tasks. In line with the Bayesian view on perception (e.g., Norris \& McQueen, 2008), we think that it is crucial to use prior knowledge in interpreting the world. The framework still allows for language to influence how we interpret and react to the world in an online and interactive manner in line with the claim of Winawer et al. (2007). But this influence does not overwrite perceptual evidence. As Norris and colleagues (Norris et al., 2000; Norris, McQueen, \& Cutler, 2003) pointed out, this is beneficial for optimal perception, because world knowledge can then be used to overcome the inherent ambiguity in perception-not only for a given stimulus, but also for the functional recalibration of categories. Several recent articles have shown how higher level knowledge is used to recalibrate perception-for example, when there is a mismatch between expected and observed perceptual information (Mitterer \& de Ruiter, 2008; Norris, Butterfield, McQueen, \& Cutler, 2006; Norris et al., 2003; van der Linden \& Vroomen, 2007). If perceptual information were to be overwritten, the mismatch between expected and observed information would go unnoticed and no recalibration could occur.

In conclusion, we set out to investigate whether declarative knowledge alone is able to influence perceptual decisions. Our data show that it can. Moreover, our data indicate that perceptual knowledge may not have an independent contribution to objectbased biases in perception. However, to conclusively demonstrate this, future researchers need to explore situations that are the mirror image of the one investigated here, where perceptual memory differs between groups but declarative knowledge remains the same.

\section{References}

Baayen, H. R., Davidson, D. J., \& Bates, D. M. (2008). Mixed-effects modeling with crossed random effects for subjects and items. Journal of Memory and Language, 59, 390-412.

Blaxton, T. (1999). Cognition: Memory, 2: Conceptual and perceptual memory. American Journal of Psychiatry, 156, 1676.

Brainard, D. H. (2004). Color constancy. In L. Chalupa \& J. Werner (Eds.), The visual neurosciences (pp. 948-961). Cambridge, MA: MIT Press.

Davidoff, J. (2001). Language and perceptual categorisation. Trends in Cognitive Sciences, 5, 382-387.

Davidoff, J., Goldstein, J., \& Roberson, D. (2009). Nature versus nurture: The simple contrast. Journal of Experimental Child Psychology, 102, $246-250$.

Deutsches Institut für Normierung, e. V. (2006). DIN EN 12368: Anlagen zur Verkehrssteuerung-Signalleuchten [Traffic control equipmentSignal heads]. Berlin, Germany: Beuth.

Drivonikou, G. V., Kay, P., Regier, T., Ivry, R. B., Gilbert, A. L., Franklin, A., \& Davies, I. R. (2007). Further evidence that Whorfian effects are stronger in the right visual field than the left. Proceedings of the National Academy of Sciences of the USA, 104, 1097-1102.

Elman, J. L., \& McClelland, J. L. (1988). Cognitive penetration of the mechanisms of perception: Compensation for coarticulation of lexically restored phonemes. Journal of Memory and Language, 27, 143-165.

Franklin, A., Wright, O., \& Davies, I. R. L. (2009). What can we learn from toddlers about categorical perception of color? Comments on Goldstein, Davidoff, and Roberson. Journal of Experimental Child Psychology, 102, 239-245. 
Frauenfelder, U. H., \& Peeters, G. (1998). Simulating the time-course of spoken word recognition: An analysis of lexical competition in TRACE. In J. Grainger \& A. M. Jacobs (Eds.), Localist connectionist approaches to human cognition (pp. 101-146). Hillsdale, NJ: Erlbaum.

Ganong, W. F. (1980). Phonetic categorization in auditory word perception. Journal of Experimental Psychology: Human Perception and Performance, 6, 110-125.

Gilbert, A. L., Regier, T., Kay, P., \& Ivry, R. B. (2006). Whorf hypothesis is supported in the right visual field but not the left. Proceedings of the National Academy of Sciences of the, USA, 103, 489-494.

Goldstein, J., Davidoff, J., \& Roberson, D. (2009). Knowing color terms enhances recognition: Further evidence from English and Himba. Journal of Experimental Child Psychology, 102, 219-238.

Hansen, T., Olkkonen, M., Walter, S., \& Gegenfurtner, K. R. (2006). Memory modulates color appearance. Nature Neuroscience, 9, 13671368.

Huettig, F., \& Altmann, G. T. M. (2004). The online processing of ambiguous and unambiguous words in context: Evidence from headmounted eye-tracking. In M. Carreiras \& C. Clifton (Eds.), The on-line study of sentence comprehension: Eyetracking, ERPs and beyond (pp. 187-207). New York, NY: Psychology Press.

Jaeger, T. F. (2008). Categorical data analysis: Away from ANOVAs (transformation or not) and towards logit mixed models. Journal of Memory and Language, 59, 434-446.

Kay, P., \& Regier, T. (2006). Language, thought and color: Recent developments. Trends in Cognitive Sciences, 10, 51-54.

Levin, D. T., \& Banaji, M. R. (2006). Distortions in the perceived lightness of faces: The role of race categories. Journal of Experimental Psychology: General, 135, 501-512.

Levinson, S. C., Kita, S., Haun, D. B. M., \& Rasch, B. H. (2002). Returning the tables: Language affects spatial reasoning. Cognition, 84, 155-188.

Li, P., \& Gleitman, L. (2002). Turning the tables: Language and spatial reasoning. Cognition, 83, 265-294.

Liberman, A. M. (1996). Speech: A special code. Cambridge, MA: MIT Press.

Magnuson, J. S., McMurray, B., Tanenhaus, M. K., \& Aslin, R. N. (2003). Lexical effects on compensation for coarticulation: A tale of two systems? Cognitive Science, 27, 801-805.

Majid, A., Bowerman, M., Kita, S., Haun, D. B. M., \& Levinson, S. C. (2004). Can language restructure cognition? The case for space. Trends in Cognitive Sciences, 8, 108-114.

Mann, V. A., \& Repp, B. H. (1981). Influence of preceding fricative on stop-consonant perception. Journal of the Acoustical Society of America, 69, 548-558.

Massaro, D. W. (1998). Perceiving talking faces: From speech perception to a behavioral principle. Cambridge, MA: MIT Press.

McClelland, J. L., \& Elman, J. L. (1986). The TRACE model of speech perception. Cognitive Psychology, 18, 1-86.

McClelland, J. L., \& Rumelhart, D. E. (1981). An interactive activation model of context effects in letter perception: I. An account of basic findings. Psychological Review, 88, 375-407.

McQueen, J. M. (2003). Letter to the editor: The ghost of Christmas future: Didn't Scrooge learn to be good? Commentary on Magnuson, McMurray,
Tanenhaus, and Aslin (2003). Journal of Memory and Language, 27, 755-759.

McQueen, J. M., Jesse, A., \& Norris, D. (2009). No lexical-prelexical feedback during speech perception or: Is it time to stop playing those Christmas tapes? Journal of Memory and Language, 61, 1-18.

Mitterer, H. (2007). Top-down effects on compensation for coarticulation are not replicable. Paper presented at the 8th Annual Conference of the International Speech Communication Association, Antwerp, Belgium.

Mitterer, H., \& de Ruiter, J. P. (2008). Recalibrating color categories using world-knowledge. Psychological Science, 19, 629-634.

Norris, D., Butterfield, S., McQueen, J. M., \& Cutler, A. (2006). Lexically guided retuning of letter perception. Quarterly Journal of Experimental Psychology, 59, 1505-1515.

Norris, D., \& McQueen, J. M. (2008). Shortlist B: A Bayesian model of continuous speech recognition. Psychological Review, 115, 357-395.

Norris, D., McQueen, J. M., \& Cutler, A. (2000). Merging information in speech recognition: Feedback is never necessary. Behavioral and Brain Sciences, 23, 299-324.

Norris, D., McQueen, J. M., \& Cutler, A. (2003). Perceptual learning in speech. Cognitive Psychology, 47, 204-238.

Pitt, M. A., \& McQueen, J. M. (1998). Is compensation for coarticulation mediated by the lexicon? Journal of Memory and Language, 39, 347370.

Presentation (Version 11.26) [Computer software]. Albany, CA: Neurobehavioral Systems. Available from www.neurobs.com

Roberson, D., \& Davidoff, J. (2000). The categorical perception of colors and facial expressions: The effect of verbal interference. Memory \& Cognition, 28, 977-986.

Roberson, D., Davies, I. R. L., \& Davidoff, J. (2000). Color categories are not universal: Replications and new evidence from a Stone-Age culture. Journal of Experimental Psychology: General, 129, 369-398.

Roberson, D., Pak, H. S., \& Hanley, J. R. (2008). Categorical perception of colour in the left and right visual field is verbally mediated: Evidence from Korean. Cognition, 107, 752-762.

Samuel, A. G., \& Pitt, M. A. (2003). Lexical activation (and other factors) can mediate compensation for coarticulation. Journal of Memory and Language, 48, 416-434.

Squire, L. R. (2004). Memory systems of the brain: A brief history and current perspective. Neurobiology of Learning and Memory, 82, 171177.

van der Linden, S., \& Vroomen, J. (2007). Recalibration of phonetic categories by lipread speech versus lexical information. Journal of Experimental Psychology: Human Perception and Performance, 33, 1483-1494.

Warren, R. M. (1970). Perceptual restoration of missing speech sounds. Science, 167, 392-393.

Winawer, J., Witthoft, N., Frank, M., Wu, L., Wade, A., \& Boroditsky, L. (2007). Russian blues reveal effects of language on color discrimination. Proceedings of the National Academy of Sciences of the USA, 107, $7780-7785$

Received September 4, 2008

Revision received May 14, 2009

Accepted June 11, 2009 